\title{
Split hand/foot malformation with long-bone deficiency and BHLHA9 duplication: A prenatal diagnosis report
}

\author{
In Ae Cho ${ }^{1}$, Ji Kwon Park ${ }^{1,2}$, Jong Chul Baek ${ }^{1, *}$, A Na Ha ${ }^{1}$, Min Young Kang ${ }^{1}$, Jae Ik Lee, Ji Eun Park ${ }^{1}$, Jeong Kyu Shin ${ }^{1,2}$, \\ Won Jun $\mathrm{Choi}^{1,2}$, Soon Ae Lee ${ }^{1,2}$, Jong Hak Lee ${ }^{1,2}$, and Won Young Paik ${ }^{1,2}$ \\ ${ }^{1}$ Department of Obstetrics and Gynecology, Gyeongsang National University School of Medicine, Jinju, Korea \\ ${ }^{2}$ Institute of Health Science, Gyeongsang National University School of Medicine, Jinju, Korea
}

Distal limb deformities are congenital malformations with phenotypic variability and high genetic heterogeneity. Split hand/ foot malformation, also known as ectrodactyly, is a congenital limb malformation characterized by a defect of the central rays of the hands and/or feet. Split hand/foot malformation with long-bone deficiency (SHFLD) is a rare condition related to a 17p13.3 duplication. Recently, genomic duplications encompassing BHLHA9 have been associated with SHFLD. We report a case of SHFLD presenting with campomelia of the right femur, bilateral agenesis of fibulae, bilateral club feet, and oligosyndactyly of the hands and feet, that was associated with a 17p13.3 duplication, as determined prenatally using array comparative genomic hybridization.

Key words: Split-hand-foot malformation with long bone deficiency, 17p13.3, BHLHA9, Comparative genomic hybridization.

\section{Introduction}

Split hand/foot malformation (SHFM) is a rare limb deformity with a prevalence of 1 in 8,500-25,000 live births that is caused by abnormal developmental signaling during embryogenesis [1]. SHFM with long-bone deficiency (SHFLD) is characterized by SHFM with the addition of long-bone deficiency usually affecting the tibia [2]. Fibular aplasia with SHFM is a rare disorder that shares several characteristics with the better known tibial aplasia-SHFM syndrome [3]. SHFLD is inherited as an autosomaldominant trait with variable expression patterns and reduced penetrance [4]. The association of a major locus on 17p13.3 was identified via linkage analysis of a large family with SHFLD $[5,6]$. The minimal 17p13.3 critical region encompasses a single gene, BHLHA9. Genomic duplication of BHLHA9 is associated with
SHFLD [2]. In this study, we report a case of SHFLD associated with a 17p13.3 duplication within BHLHA9 that was prenatally diagnosed using array comparative genomic hybridization (array (GH).

\section{Case}

\section{Case description}

A 23-year-old nulliparous woman was referred for fetal level II sonography owing to abnormal ultrasound findings, including skeletal anomalies and oligohydramnios at 20 weeks of gestation. Detailed evaluation of the fetus was difficult due to the absence of amniotic fluid. Thus, we infused normal saline into the amniotic cavity after amniocentesis for fetal karyotyping and further genetic examination. Ultrasonography

Received: 26 November 2015, Revised: 7 December 2015, Accepted: 8 December 2015, Published: 31 December 2015

*Corresponding author: Jong Chul Baek, M.D.

Department of Obstetrics and Gynecology, Gyeongsang National University School of Medicine, 15 Jinju-daero 816beon-gil, Jinju 52727, Korea.

Tel: +82-55-750-8152, Fax: +82-55-759-1118, E-mail: gnuhobgy@gmail.com

Conflict of interest: The authors declare that they do not have any conflicts of interest.

(C) This is an open-access article distributed under the terms of the Creative Commons Attribution Non-Commercial License (http://creativecommons.org/licenses/by-nc/4.0/) which permits unrestricted non-commercial use, distribution, and reproduction in any medium, provided the original work is properly cited.

(c) Copyright 2015 by the Korean Society of Medical Genetics 
revealed a normally grown fetus; biometric data of the head and abdomen were compatible with the age of gestation. Visualization of the upper limbs showed malformations with oligosyndactyly of the bilateral hands. Examination of the lower limbs demonstrated abnormal posture of flexion contractures of the knees, agenesis of fibulae, club-feet appearance, and oligosyndactyly (Fig. 1). On the following day, we carried out an ultrasonographic evaluation for fetal well-being, and the scanning view revealed severe oligohydramnios. After extensive parental counseling, the decision was made to terminate the pregnancy. The findings on the postmortem external examination and $\mathrm{X}$-rays were similar to the prenatal findings on ultrasonography (Figs. 2 and 3). Neither she nor her husband showed skeletal abnormalities, nor did any family member. We recommended the patient for parental cytogenetic evaluations; however, she refused further examination.

A

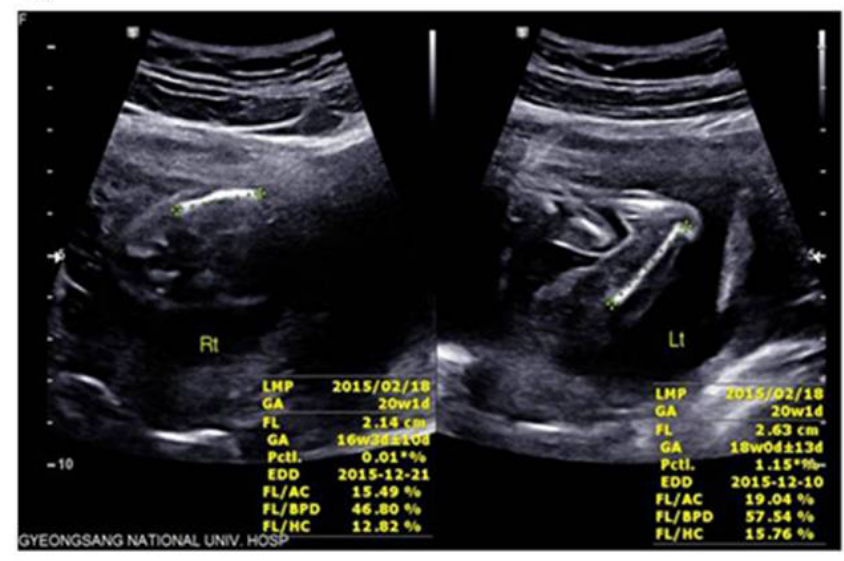

C

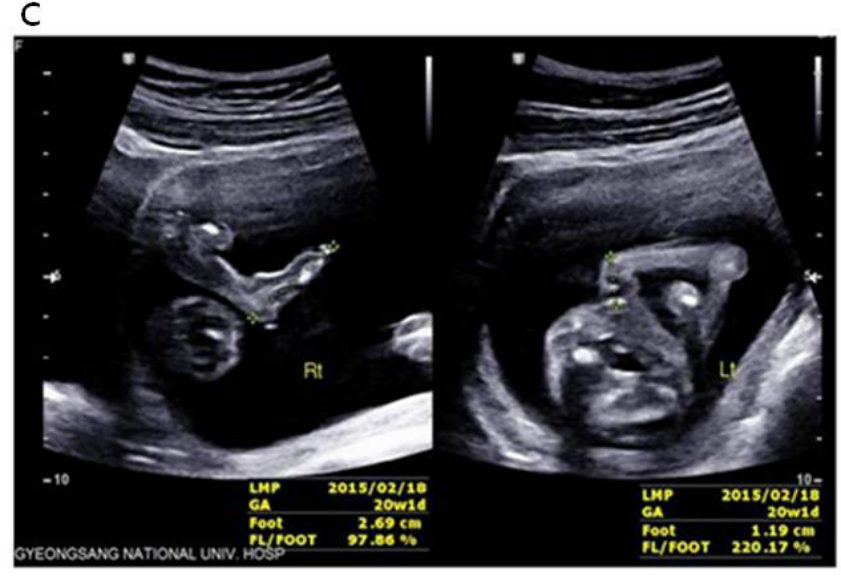

\section{Cytogenetic and $\mathrm{CGH}$ analysis}

We performed ultrasonography-guided amniocentesis. The amniotic fluid sample was processed and analyzed according to a standardized protocol. Cultivation of the amniotic fluid sample for chromosomal analysis was performed according to the method for long-term cell cultures, in which the 'flask' technique was used. Commercial medium was used for amniotic fluid cultivation (Amniomed, Euroclone, Milan, Italy; Amniomax, GIBCO-Invitrogen, Carlsbad, CA, USA). Conventional GTGbanding analysis was performed on cultured amniocytes. All 20 metaphase chromosomes from the amniotic fluid sample indicated that the fetus had normal chromosomes. We reported the fetal karyotype as 46, XY.

The genomic DNAs for array CGH analysis were extracted from amniotic fluid. The $\mathrm{CGH}$ procedure was performed as described by Kallioniemi et al. [7] and analyzed by the Cytoscan 750 K Array (Affymetrix, Santa Clara, CA, USA). The CGH profile

\section{B}

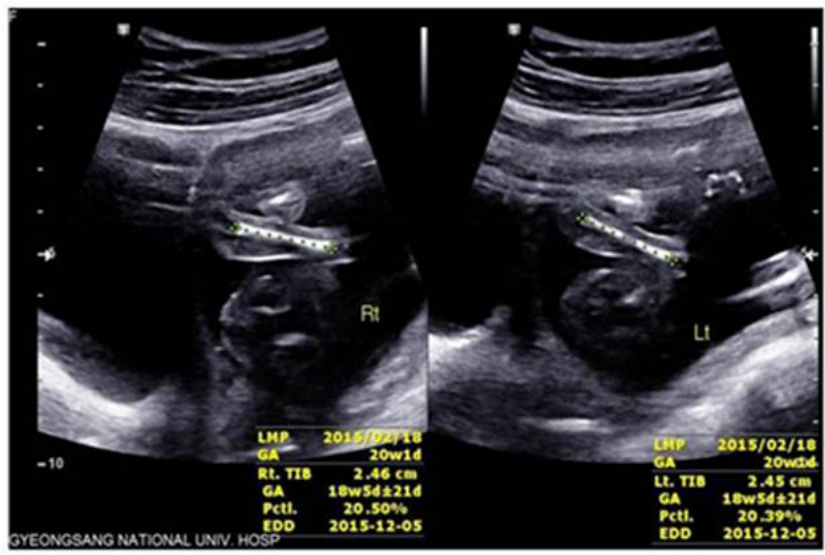

D

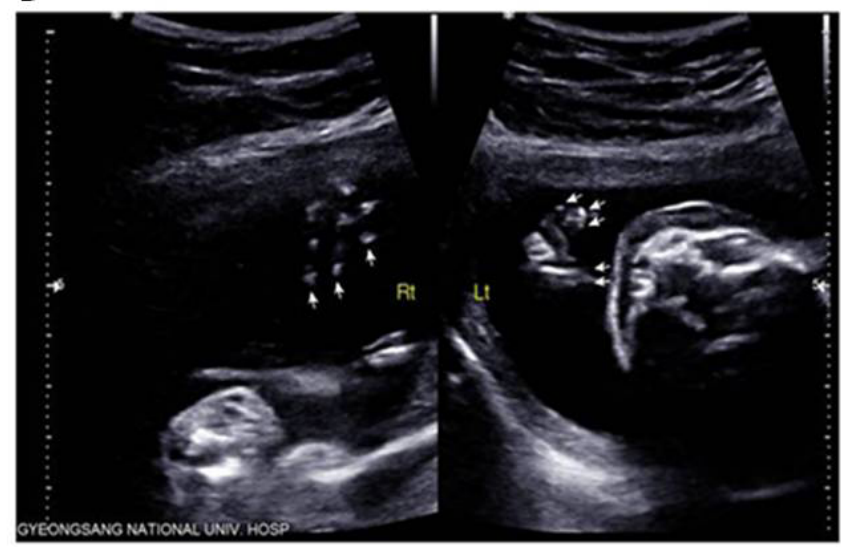

Fig. 1. High-resolution ultrasonography. (A) Right femoral campomelia. (B) Bilateral fibular agenesis. (C) Persistent flexion of the knees; club feet with oligodactyly. (D) Oligosyndactyly of the hands. 
A

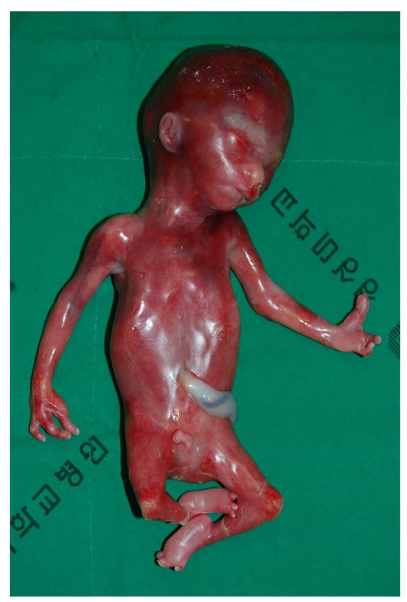

B

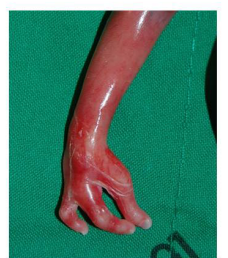

D

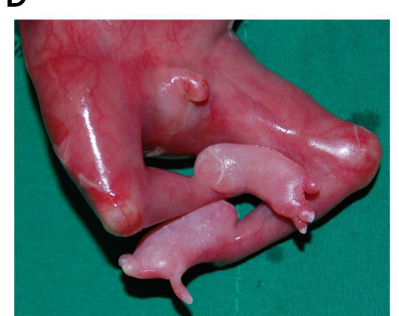

Fig. 2. Postmortem external examination. (A) Micrognathia. (B) Oligosyndactyly, right hand. (C) Oligosyndactyly, left hand. (D) External genitalia of the male, contracture of both knees, club feet, oligodactyly of both feet, and rocker-bottom feet.

C

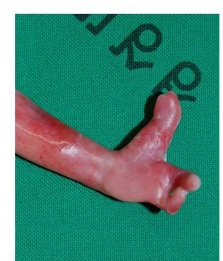

A

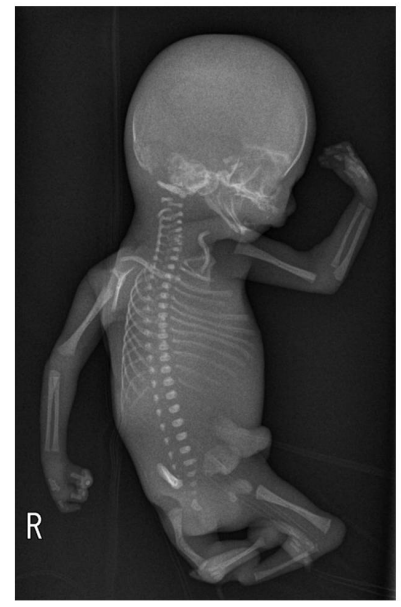

B

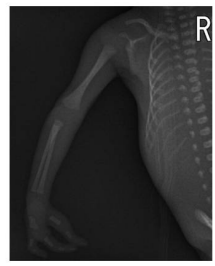

D

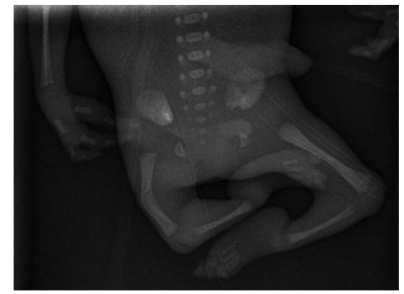

Fig. 3. Postmortem simple X-ray examination. (A) Whole-body features. (B) Oligosyndactyly, right hand. (C) Oligosyndactyly, left hand. (D) Right femoral campomelia, bilateral fibular agenesis, contracture of both knees, club feet, and oligodactyly of both feet.
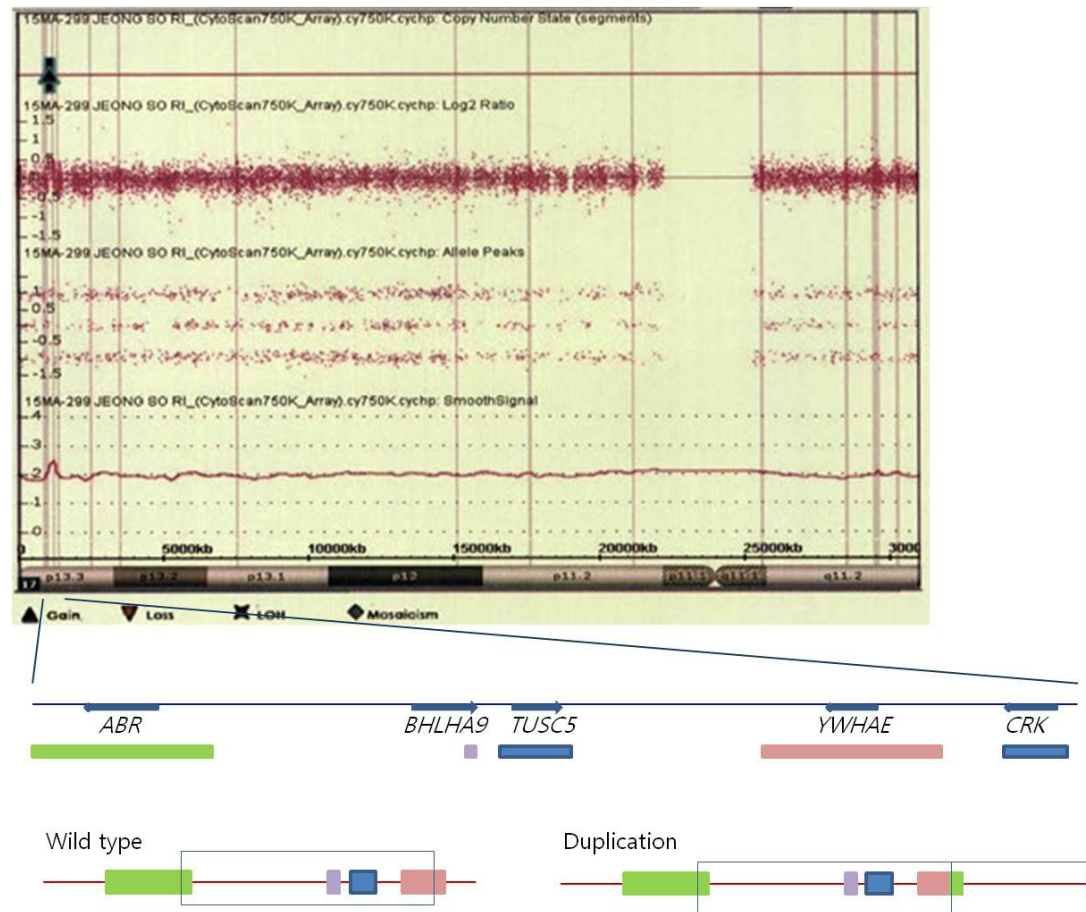

Duplication

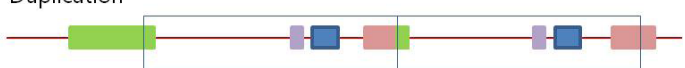

Fig. 4. Comparative genomic hybridization analysis. A 211-kb gain of signal on $17 \mathrm{p} 13.3$ associated with $A B R, B H L H A 9$, TUSC5, YWHAE, and CRK genes. showed prominent amplification of the signal in the region of 17p13.3. We identified a duplication of $211 \mathrm{~kb}$ on chromosome $17 p 13.3$ (Fig. 4). The critical genes in this region, $A B R, B H L H A 9$, TUSC5, YWHAE, and CRK, were reported. In particular, BHLHA9 is associated with SHFLD, and YWHAE is associated with developmental delay. The other genes did not present clear functions.

\section{Discussion}

Limb development during embryogenesis is a highly complex process. Deformations of the distal limbs are congenital abnormalities associated with variable phenotypes and high genetic heterogeneity. SHFM, also known as ectrodactyly, is a limb abnormality characterized by a congenital defect involving the central rays of hands and/or feet that is caused by failure to preserve the central region of the apical ectodermal ridge in the 
developing autopod $[1,8]$. SHFM is considered to originate from abnormal developmental signals during limb morphogenesis. The non-syndromic form of SHFM can be isolated (type I) or associated with long bone deficiency (type II, SHFLD), which may also present with other manifestations [1].

The known SHFM loci are associated with chromosomal rearrangements, including small duplications or deletions, of the human genome. These include SHFM1, which is associated with mutations to the $D L X 5 / 6$ gene on chromosome $7 q 21$ [9], SHFM2 on Xq26 [1], SHFM3 associated with heterozygous duplications on 10q24, the SHFM4 mutation to the TP63 locus on $3 q 27$, SHFM 5 on 2q31, and the SHFM 6 mutation to the WNT10B locus on 12q13 [1]. SHFM1 is associated with hearing loss in 35\% of patients, while SHFM with deafness is a unique clinical condition, referred to as SHFM1D, which is very complex because of the variable clinical manifestations, irregular patterns of genetic inheritance, and unique heterogeneous genetic and molecular alterations among affected individuals $[1,10]$.

SHFM is related to other limb abnormalities, as observed in SHFLD, which commonly involves the tibia, and in GollopWolfgang complex, which is characterized by bifurcation of the femur $[1,7,11]$. Fibular agenesis with SHFM has been explained as a discrete clinical entity characterized by failure of fibular development and central clefts of the hands and/or feet [12]. Fibular aplasia with SHFM is a rare disorder that shares several characteristics with the better known tibial agenesis-SHFM syndrome [3].

SHFLD is a genetically heterogeneous disorder with variable clinical manifestations within the same family and differing degrees of penetrance among individuals [13]. Most often, the inheritance of SHFLD is autosomal dominant with reduced penetrance [2]; however, autosomal recessive inheritance has been confirmed in some cases [14] and digenic inheritance is possible [15].

At least three loci, namely SHFLD1, SHFLD2, and SHFLD3, are known to be associated with SHFLD, although specific genetic causes have not yet been identified. In a retrospective study of a large consanguineous Arabic family, both 1q42.2-q43 and $6 q 14.1$, designated as loci SHFLD1 and SHFLD2, were found to be associated with SHFLD $[15,16]$. Moreover, SHFLD3 was mapped to an 861-kb interval at 17p13.1-17p13.3 in a Brazilian family [5]. Although no causative mutation is currently known, screening for copy number variations revealed diverse genomic micro-duplications on 17p13.3 [2].

The smallest area of duplication overlap markedly narrowed the critical area for the SHFLD phenotype and includes only one complete gene (BHLHA9), along with a portion of a second gene $(A B R)$ and an assumed processed transcript [1]. BHLHA9 was recently proposed as a major candidate gene in developmental limb abnormalities. BHLHA9 duplications have been identified in clinically normal family members as well as patients with SHFM and SHFLD [11]. Variable phenotypic expression is observed in patients with SHFLD harboring a 17p13.3 duplication. The most common site of long bone involvement in $17 p$ duplication carriers is reportedly the tibia [2]. Among these carriers, malespecific bias has been associated with increased susceptibility and the clinical manifestations of males appear to be more severe than those of females [2]. However, no sex-specific bias was observed in another study [14]. Although the reason for this sex-specific bias remains unknown, the presence of intraindividual variability among affected and unaffected carriers may be due to unidentified genetic factors. Hormone-responsive promoters/enhancers or the presence of a modifier on the $X$ chromosome together with $17 p$ duplication are important factors in the expression and development of SHFLD $[1,2]$.

Once fetal structural anomalies are identified by ultrasonography, prenatal chromosomal microarray analysis is useful to identify major chromosomal aneuploidy as well as submicroscopic abnormalities that could go undetected by conventional karyotyping [17]. Although the use of chromosomal microarray analysis in prenatal diagnosis remains controversial, array CGH is a useful genetic testing method for the detection of these changes as well as other unbalanced chromosomal aberrations, such as submicroscopic rearrangements affecting different foci [18]. Chromosomal microarray analysis, in addition to the ability to detect almost all abnormalities that are identifiable by fetal karyotyping, allows clinicians to identify specific genetic diseases, such as SHFM [17]. In a prenatal setting, aCGH is informative and beneficial in most cases [18]. However, the genetic and clinical heterogeneity of SHFM render genetic testing and counseling especially challenging [1]. Informed consent and genetic counseling should be addressed before the patients undergo testing.

In this report, we identified a 17p13.3 duplication containing BHLHA9 in a patient with ectrodactyly that was inherited from an unaffected parent.

\section{References}

1. Gurrieri F, Everman DB. Clinical, genetic, and molecular aspects of split-hand/foot malformation: an update. Am J Med Genet A 2013; 161A:2860-72. 
2. Klopocki E, Lohan S, Doelken SC, Stricker S, Ockeloen CW, Soares Thiele de Aguiar R, et al. Duplications of BHLHA9 are associated with ectrodactyly and tibia hemimelia inherited in non-Mendelian fashion. J Med Genet 2012;49:119-25.

3. Evans $J A$, Reed $M H$, Greenberg CR. Fibular aplasia with ectrodactyly. Am J Med Genet 2002;113:52-8.

4. Majewski F, Küster W, ter Haar B, Goecke T. Aplasia of tibia with splithand/split-foot deformity. Report of six families with 35 cases and considerations about variability and penetrance. Hum Genet 1985;70: 136-47.

5. Lezirovitz K, Maestrelli SR, Cotrim NH, Otto PA, Pearson PL, MingroniNetto RC. A novel locus for split-hand/foot malformation associated with tibial hemimelia (SHFLD syndrome) maps to chromosome region 17p13.1-17p13.3. Hum Genet 2008;123:625-31.

6. Richieri-Costa A, Brunoni D, Laredo Filho J, Kasinski S. Tibial aplasiaectrodactyly as variant expression of the Gollop-Wolfgang complex: report of a Brazilian family. Am J Med Genet 1987;28:971-80.

7. Kallioniemi A, Kallioniemi OP, Piper J, Tanner M, Stokke T, Chen L, et al. Detection and mapping of amplified DNA sequences in breast cancer by comparative genomic hybridization. Proc Natl Acad Sci U S A 1994;91:2156-60.

8. Duijf $\mathrm{PH}$, van Bokhoven $\mathrm{H}_{1}$ Brunner HG. Pathogenesis of split-hand/ split-foot malformation. Human molecular genetics 2003;12(Suppl 1): R51-60.

9. Lango Allen $H_{1}$ Caswell $R$, Xie $W, X u X$, Wragg $C$, Turnpenny PD, et al. Next generation sequencing of chromosomal rearrangements in patients with split-hand/split-foot malformation provides evidence for DYNC1/1 exonic enhancers of DLX5/6 expression in humans. J Med Genet 2014;51:264-7.

10. Elliott AM, Evans JA. Genotype-phenotype correlations in mapped split hand foot malformation (SHFM) patients. Am J Med Genet A 2006;140:1419-27.

11. Nagata E, Kano H, Kato F, Yamaguchi R, Nakashima S, Takayama S, et al. Japanese founder duplications/triplications involving BHLHA9 are associated with split-hand/foot malformation with or without long bone deficiency and Gollop-Wolfgang complex. Orphanet J Rare Dis 2014;9:125.

12. Bieganski $T$, Jamsheer $A$, Sowinska A, Baranska D, Niedzielski $K_{\text {, }}$

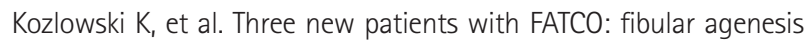
with ectrodactyly. Am J Med Genet A 2012;158A:1542-50.

13. Petit $F$, Andrieux J, Demeer $B$, Collet LM, Copin $H$, Boudry-Labis $E$, et al. Split-hand/foot malformation with long-bone deficiency and BHLHA9 duplication: two cases and expansion of the phenotype to radial agenesis. Eur J Med Genet 2013;56:88-92.

14. Witters I, Devriendt K, Moerman P, Caudron J, Van Hole C, Fryns JP. Bilateral tibial agenesis with ectrodactyly (OMIM 119100): further evidence for autosomal recessive inheritance. Am J Med Genet 2001; 104:209-13.

15. Naveed M, Nath SK, Gaines M, Al-Ali MT, Al-Khaja N, Hutchings $D$, et al. Genomewide linkage scan for split-hand/foot malformation with long-bone deficiency in a large Arab family identifies two novel susceptibility loci on chromosomes 1q42.2-q43 and 6q14.1. Am J Hum Genet 2007;80:105-11.

16. Naveed M, Al-Ali MT, Murthy SK, Al-Hajali S, Al-Khaja N, Deutsch $S$, et al. Ectrodactyly with aplasia of long bones (OMIM; 119100) in a large inbred Arab family with an apparent autosomal dominant inheritance and reduced penetrance: clinical and genetic analysis. Am J Med Genet A 2006;140:1440-6.

17. American College of Obstetricians and Gynecologists Committee on Genetics. Committee Opinion No. 581: the use of chromosomal microarray analysis in prenatal diagnosis. Obstet Gynecol 2013;122: 1374-7.

18. Sowińska-Seidler A, Badura-Stronka M, Latos-Bieleńska A, Stronka M, Jamsheer A. Heterozygous DLX5 nonsense mutation associated with isolated split-hand/foot malformation with reduced penetrance and variable expressivity in two unrelated families. Birth Defects Res $A$ Clin Mol Teratol 2014;100:764-71. 